\title{
Die Entwicklung von Sexualität bei Kindern und Jugendlichen mit sexuellen Gewalterfahrungen
}

\author{
Eine systematische Übersicht über Auswirkungen \\ auf die Sexualität
}

\author{
Katharina Louisa Schulte, Katharina Szota und Hanna Christiansen \\ Philipps-Universität Marburg
}

\begin{abstract}
Zusammenfassung: Theoretischer Hintergrund: Zu den Folgen sexueller Gewalt gehören neben somatischen und psychischen Folgen auch Veränderungen in der Entwicklung der Sexualität. Fragestellung: Ziel dieses Reviews ist es, einen systematischen Überblick über den aktuellen Forschungsstand bezüglich der Entwicklung von Sexualität bei Kindern und Jugendlichen nach sexuellen Gewalterfahrungen zu geben. Methode: Eine systematische Literaturrecherche wurde in den Datenbanken PubMed, ERIC, Cochrane und PubPsych durchgeführt. Die Folgen von sexueller Gewalt bezüglich der Sexualität wurden systematisch analysiert. Ergebnisse: Es konnten insgesamt 127 Studien zur Sexualität nach sexueller Gewalt identifiziert werden, die acht Unterkategorien zugeordnet werden konnten: Sexuelles Risikoverhalten, Teenagerschwangerschaft, jugendliche Sexualstraftäter_innen, sexuelle Verhaltensauffälligkeiten, sexuelle Überzeugungen, Prostitution, Geschlechtskrankheiten und körperliche Symptome. Diskussion und Schlussfolgerung: Insgesamt zeigt die Literaturrecherche, dass die Sexualität von Kindern und Jugendlichen mit sexuellen Gewalterfahrungen teilweise nur lückenhaft untersucht wurde.
\end{abstract}

Schlüsselwörter: sexuelle Gewalt, Sexualität, Folgen, Kinder, Jugendliche

The Development of Sexuality in Children and Adolescents with a History of Sexual Violence: A Systematic Review of the Effects on Sexuality

Abstract: Theoretical background: The effects of sexual violence in childhood are considered detrimental to and ubiquitous throughout development. In addition to physical and psychological consequences, the consequences of child sexual violence also include changes in the development of sexuality. For example, victims of child sexual violence are more likely to develop sexual distortions and sexual anxiety, engage in risky sexual behavior and transactional sex, and show sexual behavioral problems. Moreover, they are at a higher risk of becoming sexual offenders themselves. According to the current state of research, such a connection between child sexual violence and changes in sexuality has been investigated only retrospectively in adulthood after sexual violence in childhood. However, it is of great importance to better understand the aftermath of sexual violence in children and adolescents, as consequences already occur during childhood and adolescence. Objective: This review provides a systematic overview of the current state of research regarding the development of sexuality in sexually violated children and adolescents. Accordingly, the consequential question is: To what extent and in what respect does sexual violence in children and adolescents influence the sexual development of children and adolescents? Method: We carried out a systematic literature search using the following electronic databases: PubMed, ERIC, Cochrane, and PubPsych. We then analyzed the consequences of childhood sexual violence on sexuality. Results: We identified 127 studies on sexuality after sexual violence and assigned them to eight subcategories: sexual risk behavior, teenage pregnancy, juvenile sex offenders, sexual behavior problems, sexual beliefs and attitudes, transactional sex, sexually transmitted diseases, and somatic symptoms. Results show that there is a clear link between childhood sexual violence and risky sexual behavior, teenage pregnancy, transactional sex, juvenile sexual offending, sexual behavior problems, and sexual beliefs and attitudes. Discussion and conclusion: Victims of sexual violence are at a higher risk of abnormal, deviant sexual development. In children and adolescents, the development of sexuality is just beginning, making it important to intervene at an early stage to preventively reduce the effects of sexual violence. To develop appropriate interventions, the effects on sexual development need to be researched, identified, and understood. However, this research shows that to date the development of sexuality of sexually violated children and adolescents has been incompletely examined and is in need of further investigation.

Keywords: child sexual violence, consequences, sexuality, children, adolescents

Missbrauchskomplex Bergisch Gladbach, Münster und Lügde: Drei Fälle von sexueller Gewalt an Minderjähri- gen, die seit 2018 in der medialen Öffentlichkeit stehen und für Fassungslosigkeit in der Bevölkerung sorgen. Im- 
mer wieder werden Skandale sexueller Gewalt an Kindern prominent, die jedoch lediglich die Spitze des Eisbergs ausmachen.

Nach Kenntnis der polizeilichen Kriminalstatistik des Bundeskriminalamtes wurden im Jahr 2019 mehr als 15 000 Kinder Opfer sexueller Gewalt in Deutschland, wobei sich die hier berücksichtigten $\$ \$ 176,176 \mathrm{a}, 176 \mathrm{~b}$ StGB auf Betroffene unter 14 Jahren beziehen (PKS; Bundeskriminalamt, 2020). Diese Zahlen sind die polizeilich erfassten Fallzahlen. Die geschätzte Dunkelziffer liegt zwischen 1:15 (Bundeskriminalamt, 2020) und 1:20 (Kavemann \& Lohstöter, 1985), da nur jede fünfzehnte bis zwanzigste Tat angezeigt wird (Wirtz, 2001). Sogenannte Dunkelfeldforschungen schätzen, dass jeder siebte Erwachsene in Deutschland sexuelle Gewalt im Kindesalter erfahren hat (Beck, 2017).

Festzuhalten ist, dass sexuelle Gewalt ein hochrelevantes Problem ist, da bekannt ist, dass die Auswirkungen schwerwiegend sind (Noll, Trickett \& Putnam, 2003). Das Ausmaß, in dem sexuelle Gewalt Spuren hinterlässt, ist abhängig von einer Vielzahl Faktoren. Je intensiver die Tat, je häufiger sie vorgefallen ist, je länger der Zeitraum, je länger das Kind keine Hilfe bekam, je vertrauter Täter_innen sind und je weniger Verständnis das Kind erhält, desto schwerwiegender sind meistens die Konsequenzen (Beck, 2017).

Auf der einen Seite zeigen sich somatische Folgen (Pritchard \& Williams, 2010). Durch die sexuelle Gewalt selbst kann es zu leichten bis hin zu letalen Verletzungen, vor allem bei vaginaler oder analer Penetration, und einem erhöhten Infektionsrisiko kommen (Pritchard \& Williams, 2010). Aber auch chronische Erkrankungen und Schmerzzustände können langfristige Konsequenzen sein (Pritchard \& Williams, 2010).

Auf der anderen Seite lässt sich bei Personen mit sexuellen Gewalterfahrungen verglichen mit Personen mit ähnlichen Kontextbedingungen, aber ohne diese Gewalterfahrungen, ein deutlich höheres Risiko erkennen, psychisch zu erkranken (Bagley \& Mallick, 2000). In einer Langzeitstudie von Fergusson und Kollegen (1996) war das Risiko, an Depressionen zu erkranken, bei Personen mit sexuellen Gewalterfahrungen um das 3,6-fache erhöht (Fergusson, Horwood \& Lynskey, 1996). Insgesamt fanden Fergusson und Kollegen (1996) ein zwölffach erhöhtes Risiko an einer psychischen Störung nach DSM-IV zu erkranken.

Die Auswirkungen von sexueller Gewalt in der Kindheit werden demnach als schädlich für und allgegenwärtig während der gesamten Entwicklung angesehen (Noll et al., 2003). Aufgrund der explizit sexuellen Natur von sexueller Gewalt, unterscheiden sich die Auswirkungen von anderen Formen der Kindesmisshandlung darin, dass das Risiko für die Entwicklung sexueller Verzerrungen erhöht ist (Noll et al., 2003). Die aktuelle Literatur bestätigt einen solchen Zusammenhang zwischen sexueller Gewalt in der Kindheit und nachfolgenden Verzerrungen der Sexualität, einschließlich erhöhter sexueller Aktivität (z. B. Browning \& Laumann, 1997), Prostitution (z. B. Cunningham, Stiffman, Dore \& Earls, 1994; Widom \& Kuhns, 1996), früher Schwangerschaft (z. B. Fiscella, Kitzman, Cole, Sidora \& Olds, 1998), sexuellem Risikoverhalten (z.B. Brown, Kessel, Lourie, Ford \& Lipsitt, 1997) und zwanghaftem sexuellen Verhalten (z. B. Friedrich, Urquiza \& Beilke, 1986; McClellan, McCurry, Ronnei, Adams, Eisner \& Stork, 1996).

Arriola und Kolleginnen (2005) untersuchten Frauen und schlussfolgerten, dass sexuelle Gewalt an Kindern signifikant mit späterem ungeschützten Geschlechtsverkehr, Sex mit mehreren Partnern und Sexhandel (d.h. Sex gegen Geld, Drogen oder Unterkunft) zusammenhängt. Paolucci und Kollegen (2001) fanden eine Verbindung zwischen sexueller Gewalt und einer erhöhten frühzeitigen sexuellen Aktivität, Prostitution und der eigenen Beteiligung an Sexualdelikten. Whitaker und Kolleg_innen (2008) konzentrierten sich auf Risikofaktoren für die Verübung von Sexualdelikten an Kindern und stellten fest, dass Sexualstraftäter_innen mit hoher Wahrscheinlichkeit selbst missbraucht wurden im Vergleich zu anderen Personen, die entweder nicht straffällig wurden oder eine nicht-sexuell motivierte Tat verübten.

Anhand der aktuellen Studienlage kristallisiert sich heraus, dass die Folgen von sexueller Gewalt einen eminenten Einfluss auf die weitere Sexualentwicklung der Opfer haben. In der vorhandenen Literatur wird dennoch vorwiegend die Sexualität von Erwachsenen nach sexueller Gewalt in der Kindheit untersucht. Dabei sind es die Kinder und Jugendlichen, die gerade erst beginnen, die Entwicklungsaufgaben des Jugend- und frühen Erwachsenenalters zu übernehmen - eine Zeit voller Fragen der Sexualität, Identität und Intimität (Noll et al., 2003). Bislang gibt es dennoch nur eine überschaubare Anzahl an Studien, die sich mit der Entwicklung der Sexualität im Kindes- und Jugendalter nach sexueller Gewalt beschäftigt (Kendall-Tackett, Williams \& Finkelhor, 1993).

Ziel dieser Arbeit ist es, einen Überblick über den aktuellen Forschungsstand zu den Folgen von sexueller Gewalt an Kindern und Jugendlichen in Bezug auf deren Sexualentwicklung zu gewinnen. In Form eines systematischen Reviews werden verfügbare Studien zum Thema anhand festgelegter Kriterien ausgewählt und die Ergebnisse übersichtlich dargestellt. 
Tabelle 1. Suchstratgie

\begin{tabular}{|c|c|}
\hline \multirow[t]{2}{*}{ Population } & Child*, Adolescent*, Youth*, Minor*, Teen*, Juvenile*, Youngster*, Preadulthood, Young adult* \\
\hline & AND \\
\hline \multirow[t]{2}{*}{ Art der Gewalt } & Sexual* \\
\hline & AND \\
\hline \multirow[t]{2}{*}{ Gewalt } & Abuse*, Maltreatment*, Assault*, Misuse*, Misusage*, Cruelt*, Abusivene*, Violen* \\
\hline & AND \\
\hline Sexualität & $\begin{array}{l}\text { Development*, Sexual*, Drive*, Intimac*, Sensual*, Sex*, Voluptous*, Involuntary intercourse*, Voluntary intercourse*, Inter- } \\
\text { course }^{\star} \text {, Intercourse partner*, Birth control*, HIV, Risk behaviour*, Chlamydia, Gonorrhoea, Syphilis, Genital wart*, Genital herp*, } \\
\text { Hepatitis B, Hepatitis C, Pregnanc*, Abortion*, Reproductive history, Birth*, Sexual permissivene*, Sexual preoccupation*, Inter- } \\
\text { nal pressure*, External pressure*, Attitudes towards sex, Prostitut*, Sexual anxiety, Trust, Sexual distortion* }\end{array}$ \\
\hline
\end{tabular}

\section{Methode}

Dieses Review wurde vorab bei PROSPERO (https:// www.crd.york.ac.uk/prospero/) registriert und angenommen; ausführliche Information sind unter der Registrierungsnummer CRD42020145139 hinterlegt.

\section{Ein- und Ausschlusskriterien}

Für den Einschluss von Studien in das Review wurden vor der Literaturdurchsicht Kriterien festgelegt. Die Studienpopulation sollte nicht älter als 21 Jahre alt sein und sollte sexuelle Gewalterfahrungen gemacht haben. Die Studienergebnisse sollten sich zudem mit Facetten der Sexualität beschäftigen und die erfahrene sexuelle Gewalt sollte als ursächlich für die Folgen beschrieben worden sein. Nicht eingeschlossen wurden Studien, in denen sexuelle Gewalt nicht klar von anderen Misshandlungsformen differenziert oder nicht als Ursache für potentielle Folgen angesehen wurde. Einzelfallstudien wurden ausgeschlossen. Es wurden sowohl Querschnittsstudien als auch Longitudinalstudien einbezogen.

\section{Suchstrategie}

Zur Identifikation der für das Review in Frage kommenden Publikationen wurde im September 2019 eine systematische Literaturrecherche durchgeführt. Durchsucht wurden die elektronischen Datenbanken PubMed, ERIC, Cochrane und PubPsych. Es wurden Studien in englischer, deutscher oder niederländischer Sprache einbezogen. Zeitlich wurde die Suche nicht eingegrenzt. Die Literaturverzeichnisse der gefundenen Suchergebnisse wurden auf weitere relevante Studien durchgesehen. Im Rahmen der Recherche wurden verschiedene Suchbegriffe verwendet, die innerhalb der Kategorien mit „oder“ und zwischen den Kategorien mit „und“ verknüpft wurden (s. Tabelle 1).
Darauf folgte ein Screening der Titel und Abstracts anhand der Ein- und Ausschlusskriterien. In einem nächsten Schritt wurden die Volltexte der verbliebenen potentiellen Publikationen von zwei unabhängigen Ratern (KS und KS) überprüft. Dem PRISMA Flow-Chart (Moher, Liberati, Tetzlaff \& Altman, 2009) in Abbildung 1 ist die Suche und Identifikation der 127 eingeschlossenen Studien zu entnehmen.

\section{Kodierung}

Es wurden wichtige Informationen über das Studiendesign (Stichprobe, Kontrollbedingung, Datenerhebung), die Form der sexuellen Gewalt, sowie die Folgen kodiert und tabellarisch dargestellt. Die ausgewählten Studien wurden hinsichtlich der Art der Folge (Risikoverhalten, Schwangerschaft, etc.) systematisiert. Eine detaillierte Beschreibung aller 127 eingeschlossenen Artikel wird im Elektronischen Supplement 1 zur Verfügung gestellt.

\section{Ergebnisse}

Die Literaturrecherche ergab 16753 Treffer. Von diesen erfüllten 16352 Artikel nicht die Einschlusskriterien und wurden ausgeschlossen. Insgesamt 401 Studien wurden anhand des Volltextes auf Einschluss geprüft. Von diesen wurden 274 Studien ausgeschlossen, größtenteils, weil die Studienteilnehmenden älter als 21 Jahre waren $(k=61)$, keine Sexualität berichtet wurde $(k=38)$, die sexuelle Gewalt nicht Ursache sondern Folge war oder nicht in Verbindung mit dem Outcome untersucht wurde $(k=36)$ oder die sexuelle Gewalt undifferenziert erfasst wurde $(k=29)$.

Insgesamt konnten $k=127$ Studien identifiziert werden, die die Einschlusskriterien erfüllten (Abbildung 1). Diese Studien wurden zwischen den Jahren 1985 und 2019 publiziert. Die meisten Studien stammten aus den USA $(k=93)$. 


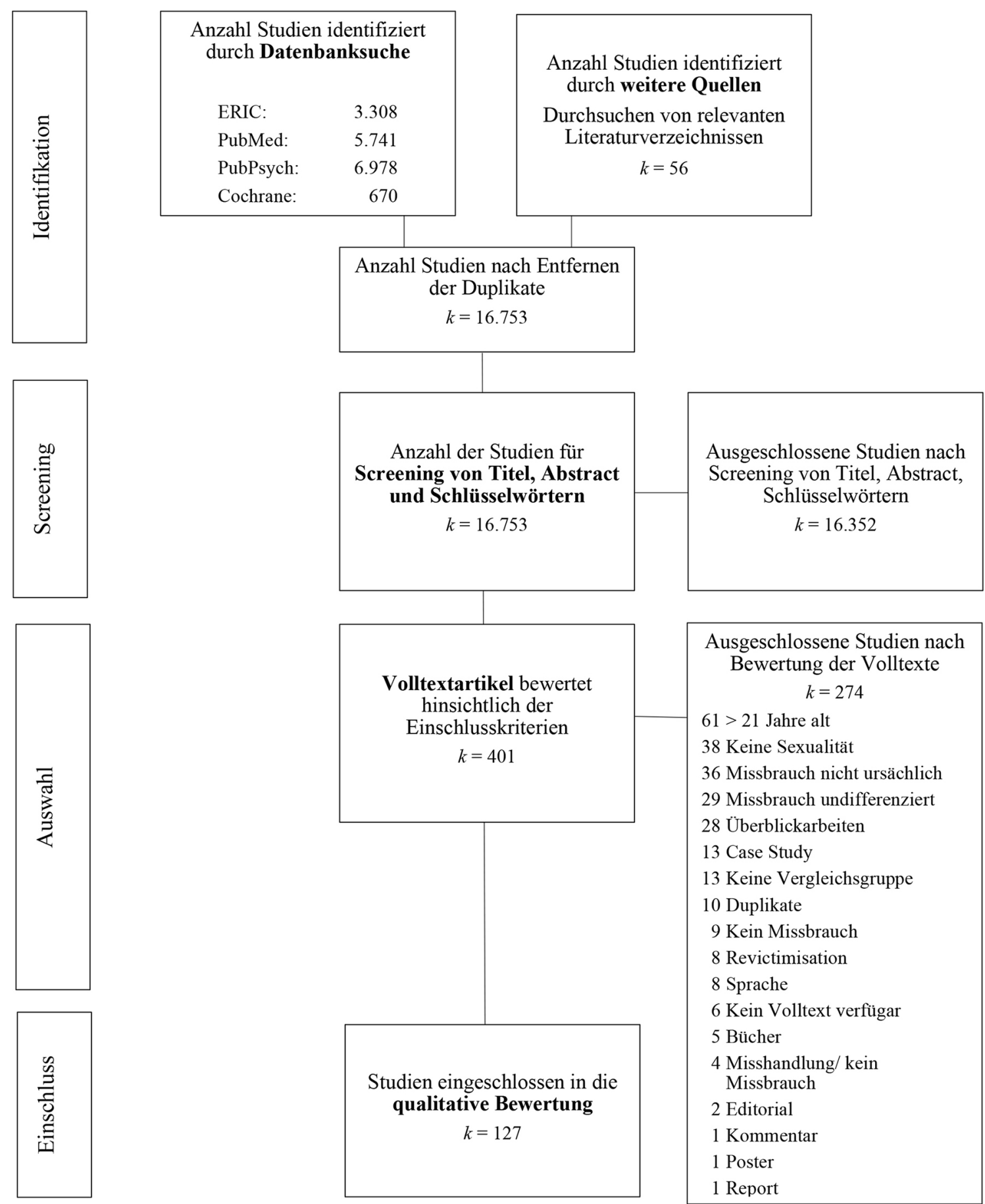

Abbildung 1. PRISMA-Flow-Diagramm zur Darstellung des Studienauswahlprozesses nach Moher, Liberati, Tetzlaff, Altman und die PRISMA Group (2009).

\section{Definition sexuelle Gewalt}

Sexuelle Gewalt wurde in den Studien auf unterschiedlichste Weise definiert: $16.5 \%(k=21)$ der Studien beschrieben sexuelle Gewalt als einen vollzogenen Sexualakt bei dem es zur Penetration (oral, anal oder vaginal) gekommen sein muss. $18.9 \%(k=24)$ sprachen von sexu- eller Gewalt, sobald es zu sexuellem Körperkontakt (,hands-on“) gekommen ist. Hierzu wurden jede Art der Penetration, aber auch Berührungen oder Küsse gezählt. Weitere $17.3 \%(k=22)$ definierten sexuelle Gewalt als jegliche sexuelle Handlung von sexueller Kommunikation („hands-off“) bis vollzogener Penetration („hands-on“). Genannt wurden unter anderem das erzwungene An- 
schauen von sexuellen Fotos oder Pornographie; Zwang, einem Erwachsene beim Onanieren zuzuschauen; einer Vergewaltigung zusehen müssen; Zwang, die Genitalien eines anderen anzufassen, etc. Bei fast der Hälfte der Studien $(47,3 \%, k=60)$ fehlte jedoch eine Definition. Diese Heterogenität sollte bei der Bewertung der Ergebnisse in Betracht gezogen werden.

\section{Stichprobe}

Insgesamt bestand die Stichprobe aus 433626 Kindern und Jugendlichen mit einem Mittelwert von 2022 und einem Median von 499 Teilnehmenden. Die kleinste Stichprobe bestand aus 40 Kindern und Jugendlichen (Orr \& Downes, 1985), die größte aus mehr als 72000 (Saewyc, Taylor, Homma \& Ogilvie, 2008). Das Alter der Kinder und Jugendlichen lag zwischen 2 und 21.6 Jahren, mit einem Durchschnittsalter von $15.6(k=68)$. Die durchschnittliche Altersspanne lag zwischen 11.2 und 17.5 $(k=102)$. Das Geschlechterverhältnis variierte deutlich in den unterschiedlichen Studien von 0 bis $100 \%$ Mädchen mit einem Mittelwert von $60.4 \%(k=93)$ weiblicher Teilnehmenden.

\section{Beschreibung der Folgen von sexueller Gewalt an Kindern und Jugendlichen}

Die Studien unterschieden sich stark bezüglich der Population und der Folgen der sexuellen Gewalt. Um der Heterogenität der Studien entgegenzuwirken, wurden die Folgen sexueller Gewalt acht Unterkategorien zugeordnet: Sexuelles Risikoverhalten, Teenagerschwangerschaft, jugendliche Sexualstraftäter_innen, sexuelle Verhaltensauffälligkeiten, sexuelle Überzeugungen und Einstellungen, Prostitution, Geschlechtskrankheiten und körperliche Symptome.

\section{Sexuelles Risikoverhalten}

Mit einer Anzahl von 58 Studien ist sexuelles Risikoverhalten die am häufigsten untersuchte Folge. Am häufigsten wurde die Anzahl der Sexualpartner untersucht $(k=$ 31 ), gefolgt von der Benutzung von Verhütungsmitteln ( $k$ $=26)$, dem Alter beim ersten Koitus $(k=22)$ und dem Geschlechtsverkehr unter dem Einfluss von Alkohol und Drogen $(k=11)$. Andere Aspekte, zu denen ein positiver Zusammenhang gefunden wurde, waren unter anderem Geschlechtsverkehr mit Hochrisikopartnern (Wilson, Emerson, Donenberg \& Pettineo, 2013) oder Fremden (Carlson, Oshri \& Kwon, 2015) oder als selbstverletzendes Verhalten (Zetterqvist, Svedin, Fredlund, Priebe, Wadsby \& Jonsson, 2018). Im Durchschnitt waren die Studienteil- nehmer_innen 15.5 Jahre alt und überwiegend weiblich (Durchschnitt 87.6\%). Insgesamt fanden $90 \%$ der Studien $(k=52)$ einen positiven Zusammenhang zwischen erlebter sexueller Gewalt und sexuellem Risikoverhalten.

\section{Teenagerschwangerschaft}

Eine weitere Folge von sexueller Gewalt sind Teenagerschwangerschaften. Insgesamt befassten sich dreißig Studien mit Schwangerschaften, von denen $67 \%(k=20)$ ein erhöhtes Risiko nach sexuellen Gewalterfahrungen fanden. Im Durchschnitt waren $85 \%(k=22)$ der Befragten weiblich. Pierre et al. (1998) untersuchten männliche Jugendliche und fanden ein erhöhtes Risiko zur Schwangerschaftsbeteiligung bei Jugendlichen mit sexuellen Gewalterfahrungen (Pierre, Shrier, Emans \& DuRant, 1998). Sechs Studien bestätigten diesen Zusammenhang sowohl für männliche, als auch für weibliche Studienteilnehmende (Anteghini, Fonseca, Ireland \& Blum, 2001; Nagy, Adcock \& Nagy, 1994; Trent, Clum \& Roche, 2007; Raj, Silvermann \& Amaro, 2000; Saewyc, Magee \& Pettingell, 2004; Saewyc et al., 2008). Sechs weitere Studien untersuchten weibliche Stichproben und fanden ebenfalls eine positive Korrelation zwischen sexueller Gewalt und Teenagerschwangerschaften (Cinq-Mars, Wright, Cyr \& McDuff, 2004; Noll \& Shenk, 2013; Noll et al., 2019; Ochen, Chi \& Lawoko, 2019; Thrane \& Chen, 2012; Chandy, Blum \& Resnik, 1996). Cinq-Mars et al. (2004) fanden ein dreizehnfach erhöhtes Risiko einer Teenagerschwangerschaft, wenn eine Penetration stattgefunden hat (Cinq-Mars et al., 2004).

\section{Jugendliche Sexualstraftäter_innen}

Sexuelle Straftaten wurden als eine weitere Folge sexueller Gewalt genannt. Dieser Unterkategorie ließen sich 26 Studien zuordnen. Insgesamt $77 \%(k=20)$ fanden einen positiven Zusammenhang zwischen sexueller Gewalt und Verstößen gegen das Sexualstrafrecht. Untersucht wurde diese Verbindung überwiegend bei männlichen Studienteilnehmenden (Durchschnitt 5.9\% weibliche Teilnehmende, $k=15)$. Das Durchschnittsalter der Teilnehmenden betrug 15.8 Jahre $(k=17)$. Zwölf Studien analysierten Fallberichte zur Datenerhebung, 17 führten Interviews oder Surveys durch. Insgesamt wurden überwiegend straffällig gewordene Jugendliche in Vollzugsanstalten oder in forensisch-psychiatrischen Unterbringungen befragt.

Aebi et al. (2015) fanden einen positiven Zusammenhang zwischen sexueller Gewalt mit und ohne physischen Kontakt und grenzüberschreitendem sexuellen Verhalten. Barra et al. (2017) fanden eine positive Korrelation für sexuelle Gewalt in der Adoleszenz, nicht aber für das Kindesalter. Bladon et al. (2005) untersuchten 141 männliche straffällig gewordene Jugendliche und fanden her- 
aus, dass $71 \%$ sexuelle Gewalt in ihrer Kindheit oder Jugend erfahren hatten. Borowsky und Kolleginnen (1997) bestätigten einen positiven Zusammenhang, differenzierten aber nach Status des Täters/der Täterin (Familienmitglied versus Erwachsener außerhalb der Familie); in beiden Fällen zeigt sich eine positive Korrelation.

\section{Sexuelle Überzeugungen und Einstellungen}

Die Literatursuche ergab 17 Studien, die sich kategorisch den sexuellen Überzeugungen und Einstellungen zuordnen ließen. Alle Studien stammten aus den USA. Rund siebzig Prozent $(k=12)$ der Studien berichteten einen signifikanten Zusammenhang zwischen sexueller Gewalt und verschiedenen sexuellen Überzeugungen (sexuelle Angst, Selbstakzeptanz, Diskomfort, etc.). In diesen Studien wurde ein signifikanter positiver Zusammenhang zwischen sexueller Gewalt und sexueller Angst gefunden (Cohen, Deblinger, Maedel \& Staffer, 1999; Feiring, Taska \& Lewis, 1999; Feiring, Taska \& Lewis, 1998; Simon \& Feiring, 2008), wobei Feiring et al. (1998) diese Korrelation nur bei zusätzlicher Anwendung körperlicher Gewalt bestätigen konnten. Esparza und Esperat (1996) fanden eine Verbindung zwischen sexuellen Gewalterfahrungen und reduzierter sexueller Selbstakzeptanz. Positive Korrelationen wurden darüber hinaus zwischen sexueller Gewalt und sexuellen Bedenken (Dillard, Maguire-Jack, Showalter et al., 2019; Friedrich, Jaworski, Huxsahl \& Bengtson, 1997), der Selbstwirksamkeit bei der Verwendung von Kondomen (Brown, Kessel, Lourie et al., 1997; Houck et al., 2010) oder der eigenen Geschlechtsidentifikation (Aiosa-Karpas, Karpas, Pelcovitz \& Kaplan, 1991; Rosario, Schrimshaw \& Hunter, 2008) berichtet.

\section{Sexuelle Verhaltensauffälligkeiten}

Insgesamt thematisierten 16 Studien sexuelle Verhaltensauffälligkeiten als Folge von sexueller Gewalt. Als Verhaltensauffälligkeiten wurden unter anderem hypersexuelles, sexuell übergriffiges und sexuell ängstliches Verhalten, das Entblößen von Genitalien, Erotizismus und sexuelle Aggressionen beschrieben.

Das Durchschnittsalter $(k=11)$ lag bei 9.11 Jahren. Untersucht wurden überwiegend jüngere Kinder ab einem Alter von 2 Jahren. Aufgrund des Alters der Probanden wurde in $87.5 \%$ der Studien die Datenerhebung auf Basis von Fremdbeurteilungen durchgeführt. Dreiviertel der Studien fanden einen signifikanten Zusammenhang zwischen sexueller Gewalt und sexuellen Verhaltensauffälligkeiten. Sowohl Cole et al. (2016), als auch Hall et al. (1998) fanden eine signifikant positive Verbindung zwischen sexueller Gewalt und entwicklungsbedingt unangemessenem, sexualisiertem Verhalten. Cosentino und et al. (1995) untersuchten sexuelle Aggression bei 6- bis 12jährigen Mädchen und fanden ein signifikant höheres
Ausmaß sexueller Aggression bei Kindern mit sexuellen Gewalterfahrungen.

Ebenfalls deutlich wurde in der Literatur, dass die Häufigkeit und Dauer der sexuellen Gewalt (siehe Feiring et al., 1999; Friedrich et al., 1986), die körperliche Erregung während der Gewalterfahrung (Hall et al., 1998) und das Alter, in dem die Gewalt begann (McClellan et al., 1996), prädiktiv für das Auftreten von sexuellen Verhaltensauffälligkeiten sind.

Feiring et al. (2009) konnten als einzige Studie keinen direkten Zusammenhang zwischen sexueller Gewalt und sexuellen Verhaltensauffälligkeiten bestätigen. Unter Berücksichtugung von missbrauchsspezifischer Stigmatisierung konnte der Zusammenhang nachgewiesen werden (Feiring et al., 2009).

\section{Geschlechtskrankheiten}

Insgesamt elf Studien befassten sich mit Geschlechtskrankheiten als Folge von sexueller Gewalt. Neun der elf Studien stammten aus den USA und sieben Studien untersuchten Geschlechtskrankheiten lediglich bei weiblichen Teilnehmenden. Untersucht wurden 13- bis 21-jährige Jugendliche mit einem Durchschnittsalter von 16.47 Jahren $(k=10)$. Ein Großteil der Studien fragte dichotom nach dem Auftreten einer Geschlechtskrankheit, wohingegen Vermund et al. (1990) sich explizit auf Gonorrhoe und Syphilis bezogen. Champion (2011) erfragte Chlamydien und Noell et al. (2001) Genitalherpes (HSV-2), Hepatitis B und Chlamydien. Sechs der elf Studien fanden eine positive Korrelation, vier Studien konnten keinen Zusammenhang finden.

\section{Prostitution}

Die systematische Literatursuche resultierte in fünf Studien, die sich mit Prostitution befassen. Ahrens et al. (2012) untersuchten 732 Jugendliche im Alter von 19 bis 21 Jahre, die außerhäuslich untergebracht waren und fanden einen positiven Zusammenhang zwischen sexueller Gewalt und transaktionalem Geschlechtsverkehr bei weiblichen Jugendlichen, die sich viermal häufiger prostituierten als außerhäusliche untergebrachte Jugendliche ohne sexuelle Gewalterfahrungen (Ahrens, Katon, McCarty et al., 2012). Rotheram-Borus et al. (1996) untersuchten ebenfalls 190 außerhäuslich lebende Jugendliche und fanden einen signifikanten Zusammenhang zwischen sexueller Gewalt und Prostitution (RotheramBorus, Mahler, Koopman \& Langabeer, 1996).

\section{Körperliche Symptome}

Insgesamt drei Studien beschrieben körperliche Symptome als Folge von sexueller Gewalt. Wu et al. (2003) fanden in ihrer Studie mit 791 weiblichen Jugendlichen (Durchschnittsalter 16.2 Jahre) einen positiven Zusam- 
menhang zwischen sexueller Gewalt und Infektionen im Becken- und Eileiterbereich. Santerre-Baillargeon et al. (2017) untersuchten genito-pelvine Schmerzen bei 218 Mädchen (Durchschnittsalter 16.2 Jahre), fanden aber keine signifikante Korrelation. Worku et al. (2006) befragten 323 weibliche Neuntklässler $\mathrm{zu}$ verschiedenen körperlichen Folgeerscheinungen. Etwa 3.2\% der Befragten mit sexuellen Gewalterfahrungen gaben an, Genitalläsionen zu haben, $2.7 \%$ beschrieben Genitalausfluss und $1.4 \%$ klagten über sexuelle Dysfunktionen.

\section{Diskussion}

Das primäre Ziel dieses Reviews war, einen Überblick über den aktuellen Forschungsstand zu den Folgen von sexueller Gewalt an Kindern und Jugendlichen in Bezug auf deren Entwicklung von Sexualität zu gewinnen und die Zusammenhänge besser zu verstehen. Die hier berichteten Ergebnissen stützen die Annahme, dass sexuelle Gewalt im Kindes- und Jugendalter ein Risikofaktor für eine abweichende sexuelle Entwicklung sein kann. Kinder und Jugendliche mit sexuellen Gewalterfahrungen berichteten signifikant häufiger von sexuellem Risikoverhalten, Teenagerschwangerschaften, sexuellen Verhaltensauffälligkeiten, Prostitution, veränderten sexuellen Überzeugungen und Verstößen gegen das Sexualstrafrecht. Aufgrund der dünnen Studienlage konnte keine Aussage über den Zusammenhang zwischen sexuellen Gewalterfahrungen und somatischen Folgen und Geschlechtskrankheiten getroffen werden.

Diese Ergebnisse stehen im Einklang mit dem 1985 aufgestellten und immer noch gültigem Traumagenic Dynamics Model of Sexual Abuse (Finkelhor \& Browne, 1985). Es ist ein Modell, das eine Vielzahl unterschiedlicher Dynamiken einbezieht, um diverse Folgen sexueller Gewalt zu berücksichtigen. Das Modell schlägt vier traumagene Dynamiken vor, um die Auswirkungen sexueller Gewalt zu erklären: traumatische Sexualisierung, Stigmatisierung, Vertrauensmissbrauch bzw. Verrat und Hilflosigkeit und Ohnmacht (Finkelhor \& Browne, 1985). Eine traumagene Dynamik ist eine Erfahrung, die die kognitive oder emotionale Orientierung eines Kindes zur Welt verändert und ein Trauma verursacht, indem es das Selbstkonzept, die Weltanschauung oder die affektiven Fähigkeiten des Kindes verzerrt (Finkelhor \& Browne, 1985). Die Dynamik der Stigmatisierung kann zum Beispiel das Gefühl für den eigenen Wert verzerren (Finkelhor \& Browne, 1985). Die Dynamik der Hilflosigkeit und Ohnmacht kann das Gefühl verändern, das eigene Leben kontrollieren zu können (Finkelhor \& Browne, 1985). Wenn eine Person mit solchen Verzerrungen folglich ver- sucht, mit der Welt zurechtzukommen, resultiert dies häufig in psychischen Problemen und Verhaltensauffälligkeiten, die für Kinder und Erwachsene mit sexuellen Gewalterfahrungen charakteristisch sind (Finkelhor \& Browne, 1985).

Bezogen auf sexuelles Risikoverhalten und Prostitution könnte dies bedeuten, dass bestimmte negative sexuelle Stigmata, die mit dem Opfer sexueller Gewalt verbunden sind, in das Selbstkonzept integriert werden, was die Bildung einer zwanghaften Replikation von bestimmten sexuellen Gefühlen und Erfahrungen begünstigt, die durch die sexuelle Gewalterfahrung gelernt wurden (Noll et al., 2003). Anschließend werden diese Wiederholungen der sexuellen Gefühle und Erfahrungen in Situationen gesucht, die den sexuellen Gewalterfahrungen ähneln, was zu einer Übergeneralisierung der sexuellen Gewalterfahrung auf andere enge Beziehungen führen kann (Noll et al., 2003). Solche überwiegend unbewussten Ansichten können eine Unfähigkeit begünstigen, die es erschwert nicht-sexuelle oder emotionale Belohnungen aus Beziehungen zu gewinnen. Dies könnte erklären, wieso sich Opfer sexueller Gewalt signifikant häufiger in potentiell riskante Situationen begeben und sich sexuell ausbeuten lassen (Noll et al., 2000). Hieraus lässt sich wiederum der positive Zusammenhang zwischen sexueller Gewalt und Geschlechtskrankheiten ableiten, da sexuelles Risikoverhalten (wechselnde Partner, ungeschützter Geschlechtsverkehr, Sex unter dem Einfluss von Alkohol/Drogen, etc.) die Wahrscheinlichkeit einer Ansteckung erhöht.

Eine weitere Erklärung für ein erhöhtes sexuelles Risikoverhalten könnten durch sexuelle Gewalterfahrungen veränderte Bindungsmuster sein. Bindungsstörungen wurden überproportional häufig bei Opfern sexueller Gewalt gefunden, die zu unsicheren oder sogar ängstlichen und vermeidenden Bindungen in dyadischen Beziehungen führen können (Kwako, Noll, Putnam \& Trickett, 2010). Eine solche Bindung kann in fehlender Kommunikation über sexuelle Bedürnnisse und Grenzen resultieren (Kwako et al., 2010) und dementsprechend Risikoverhalten, wie zum Beispiel die Nichtnutzung von Kondomen, begünstigen.

Darüber hinaus kann sich ein unsicherer Bindungsstil nicht nur in dysfunktionalen romantischen Bindungen manifestieren, sondern auch dazu führen, dass sexuelles Verhalten dazu dient, Bindungsbedürfnisse zu befriedigen und weniger durch persönliche Bedürfnisse motiviert ist (Noll et al., 2019). Bezogen auf Teenageschwangerschaften und Elternschaft könnte dies bedeuten, dass Überlebende sexueller Gewalt ein Kind als eine Art Wiedergutmachung für beschädigte Beziehungen $\mathrm{zu}$ geliebten Menschen sehen, oder dass das Mutte-/Vaterrsein eine unzerbrechliche Bindung schafft (Noll et al., 2019).

Neben der Verbindung zwischen sexuellen Gewalterfahrungen und sexuellem Risikoverhalten, Teenager- 
schwangerschaft und verzerrten sexuellen Einstellungen, wurde in der Mehrzahl der Studien ein Zusammenhang zwischen sexueller Gewalt und sexuellen Verhaltensauffälligkeiten, sowie Verstößen gegen das Sexualstrafrecht gefunden. Diese zuletzt genannten beiden Folgen sexueller Gewalt werden häufig anhand ätiologischer Theorien des sozialen Lernens erklärt (siehe Burton \& Meezan, 2004; Garland \& Dougher, 1990), wonach Kinder und Jugendliche, die Opfer sexueller Gewalt geworden sind oder viel sexuelles Verhalten beobachtet haben (in vivo, per Video oder durch andere Medien), möglicherweise wiederholen, was mit ihnen gemacht wurde oder was sie sahen (Burton, 2008). Gemäß der sozial-kognitiven Theorie, der neuesten Version von Banduras Theorie des sozialen Lernens (1986), können mehrere theoretische Pfade entweder einzeln oder in Kombination für die Entwicklung sexuell missbräuchlicher Verhaltensweisen verantwortlich sein (Burton \& Meezan, 2004).

Bezüglich somatischer Folgeerscheinungen bei Kindern und Jugendlichen durch sexuelle Gewalt kann dieser Artikel keine Aussage treffen. Lediglich drei Studien befassten sich mit somatischen Auswirkungen bezogen auf die Sexualität von Kindern und Jugendlichen. Festzuhalten ist, dass sich die Studienlage in diesem Bereich als sehr lückenhaft herausstellt. Insgesamt ist die Studienlage im Bereich der Forschung mit Kindern und Jugendlichen hinsichtlich der Auswirkungen von sexuellen Gewalterfahrungen auf die Sexualitätsentwicklung äußerst rudimentär (Kendall-Tackett, Williams \& Finkelhor, 1993).

In der vorhandenen Literatur wird überwiegend die Sexualität von Erwachsenen nach sexueller Gewalt in der Kindheit untersucht. Dabei sind es die Kinder und Jugendlichen, bei denen die Entwicklung der Sexualität gerade erst anfängt und bei denen es wichtig wäre, frühzeitig zu intervenieren, damit die Auswirkungen von sexueller Gewalt präventiv reduziert werden. Aufgrund dessen besteht eine enorme Wichtigkeit, die Folgen bereits im Kindes- und Jugendalter zu identifizieren und verstehen, um wirksame Interventionen zu entwickeln und anzubieten. Solche Interventionen könnten die Behandlung von Angstzuständen und dissoziativen Symptomen, die Umkehrung potenzieller sexueller Stigmatisierungen und den Wiederaufbau von Vertrauen und Empowerment umfassen (Noll et al., 2003).

\section{Limitationen}

Sowohl die einzelnen Studien selbst als auch dieses Review weisen einige Limitationen auf. Die größte Einschränkung dieses Reviews ist die hohe Heterogenität bezüglich der Studienpopulationen, der Methodologie und der Ergebnisse der einzelnen Studien. Diese sollte bei der Interpretation der Studienergebnisse nicht außer Acht gelassen werden. Eine weitere Einschränkung betrifft den querschnittlichen Charakter der Studien. Querschnittsstudien schränken kausale Schlussfolgerungen ein, obwohl die chronologische Reihenfolge der sexuellen Gewalt und das nachfolgende Risikoverhalten diese Limitation eingrenzen. Darüber hinaus sollten unerforschte Variablen, die mit sexueller Gewalt und riskantem Sexualverhalten zusammenhängen können (z.B. weitere traumatische Erfahrungen; Dong, Anda, Dube, Giles \& Felitti, 2003), bei zukünftigen Studien berücksichtigt werden.

Des Weiteren besteht zwischen den Studien kein deutliches und einheitliches Konzept darüber, wie sexuelle Gewalt definiert wird. Teilweise muss es zwingend $\mathrm{zu}$ Körperkontakt oder sogar zur Penetration (,hands-on') gekommen sein, damit Studien von dem Auftreten von sexueller Gewalt sprechen. In anderen Studien beginnt sexuelle Gewalt bereits schon bei Situationen ohne Körperkontakt (,hands-off'). Diese Varianz führt zu vielfältigen Problemen in Forschung, aber auch Politik, Recht, Prävention und der Gestaltung sozialer Normen (Mathews \& Collin-Vézina, 2019). Dennoch besteht allgemeiner Konsens darüber, dass sexuelle Gewalt ein komplexes Phänomen ist, das aus verschiedenen Gründen auf verschiedene Weise und in unterschiedlichen Beziehungen innerhalb von Familien, Peer-Gruppen, Institutionen und Gemeinschaften auftritt (Mathews \& Collin-Vézina, 2019). Aufgrund dessen besteht eine enorme Wichtigkeit, sexuelle Gewalt eindeutig zu definieren und operationalisieren. Die Weltgesundheitsorganisation (WHO, 2003) stellte diesen Bedarf ebenfalls fest, als sie dazu aufrief, dass die verschiedenen Sektoren, die an der Bekämpfung von sexueller Gewalt beteiligt sind, eine gemeinsame konzeptionelle Definition von sexueller Gewalt und gemeinsame operative Definitionen entwickeln müssen, um die Identifizierung von Fällen zu ermöglichen.

Eine weitere konzeptionelle Limitation ist die Sexualitätsentwicklung. Da Sexualität ein facettenreiches Konstrukt darstellt und die Entwicklung der Sexualität sehr individuell verläuft, ist es wichtig, sich in einem nächsten Schritt die jeweiligen Unterkategorien dieses Überblicksartikels genauer anzuschauen. Hierbei sollten die Definition und Art der sexuellen Gewalt, die Altersspanne und das Geschlecht der Probanden als mögliche Mediatoren in Betracht gezogen werden, um eine bessere Identifikation, Prävention und Intervention zu ermöglichen.

\section{Schlussfolgerung und Ausblick}

Dieses Review zeigt, dass sexuelle Gewalt an Kindern und Jugendliche gravierende Folgen mit sich bringt, die während der gesamten Entwicklung omnipräsent sind. Durch 
diese Arbeit wird deutlich, dass aufgrund der sexuellen Natur der Erfahrungen, sexuelle Gewalt einen signifikanten Einfluss auf die Entwicklung der Sexualität im Kindesund Jugendalter hat. Aus diesem Grunde besteht eine immense Wichtigkeit, die Folgen bereits im Kindes- und Jugendalter zu identifizieren und verstehen, um besser und frühzeitig intervenieren zu können, und um gravierende Spätfolgen (wie zum Beispiel selbst Täter_in werden) zu reduzieren.

\section{Elektronische Supplemente (ESM)}

Die elektronischen Supplemente sind mit der OnlineVersion dieses Artikels verfügbar unter https://doi.org/ 10.1026/0942-5403/a000345

ESM 1. Tabelle E1. Studienbeschreibung Die Tabelle zeigt eine detaillierte Beschreibung aller 127 Studien.

\section{Literatur}

Aebi, M., Landolt, M. A., Mueller-Pfeiffer, C., Schnyder, U., Maier, T. \& Mohler-Kuo, M. (2015). Testing the "sexually abused-abuser hypothesis" in adolescents: A population-based study. Archives of Sexual Behavior, 44, $2189-2199$.

Ahrens, K. R., Katon, W., McCarty, C., Richardson, L. P. \& Courtney, M.E. (2012). Association between childhood sexual abuse and transactional sex in youth aging out of foster care. Child Abuse \& Neglect, $36,75-80$.

Aiosa-Karpas, C. J., Karpas, R., Pelcovitz, D. \& Kaplan, S. (1991). Gender identification and sex role attribution in sexually abused adolescent females. Journal of the American Academy of Child \& Adolescent Psychiatry, 30, $266-271$.

Anteghini, M., Fonseca, H., Ireland, M. \& Blum, R. W. (2001). Health risk behaviors and associated risk and protective factors among Brazilian adolescents in Santos, Brazil. Journal of Adolescent Health, 28, 295-302.

Arriola, K. R., Louden, T., Doldren, M. A. \& Fortenberry, R. M. (2005). A meta-analysis of the relationship of child sexual abuse to HIV risk behavior among women. Child Abuse \& Neglect, 29, $725-$ 746.

Bagley, C. \& Mallick, K. (2000). Prediction of sexual, emotional, and physical maltreatment and mental health outcomes in a longitudinal cohort of 290 adolescent women. Child Maltreatment, 5 , $218-226$.

Bandura, A. (1986). Social foundations of thought and action. Englewood Cliffs, NJ, 1986, $23-28$.

Barra, S., Bessler, C., Landolt, M. A. \& Aebi, M. (2017). Type and timing of maltreatment influence criminal persistence in sexually abusive adolescents. Law and Human Behavior, 41, 556.

Beck, F. (UBSKM) (2017). Fakten und Zahlen zu sexueller Gewalt an Kindern und Jugendlichen. Unabhängiger Beauftragter für Fragen des sexuellen Kindesmissbrauchs, zuletzt geprüft am 24.06. 2019. Verfügbar unter: https://beauftrag ter-missbrauch.de
Bladon, E. M., Vizard, E., French, L. \& Tranah, T. (2005). Young sexual abusers: A descriptive study of a UK sample of children showing sexually harmful behaviours. Journal of Forensic Psychiatry \& Psychology, 16, 109-126.

Borowsky, I. W., Hogan, M. \& Ireland, M. (1997). Adolescent sexual aggression: Risk and protective factors. Pediatrics, 100, e7-e7.

Brown, L. K., Kessel, S. M., Lourie, K. J., Ford, H. H. \& Lipsitt, L. P. (1997). Influence of sexual abuse on HIV-related attitudes and behaviors in adolescent psychiatric inpatients. Journal of the American Academy of Child \& Adolescent Psychiatry, 36, 316 322.

Browning, C. \& Laumann, E. (1997). Sexual contact between children and adults. A life course perspective. American Sociological Review, 62, $540-560$.

Bundeskriminalamt (2020). Polizeiliche Kriminalstatistik 2008, Bundesrepublik Deutschland. Wiesbaden: Bundeskriminalamt.

Burton, D. L. \& Meezan, W. (2004). Revisiting recent research on social learning theory as an etiological proposition for sexually abusive male adolescents. Journal of Evidence-Based Social Work, 1, 41-80.

Burton, D. L. (2008). An exploratory evaluation of the contribution of personality and childhood sexual victimization to the development of sexually abusive behavior. Sexual Abuse, 20, $102-$ 115.

Carlson, M., Oshri, A. \& Kwon, J. (2015). Child maltreatment and risk behaviors: The roles of callous/unemotional traits and conscientiousness. Child Abuse \& Neglect, 50, 234-243.

Champion, J. D. (2011). Context of sexual risk behaviour among abused ethnic minority adolescent women. International Nursing Review, 58, $61-67$

Chandy, J. M., Blum, R. W. \& Resnick, M. D. (1996). Female adolescents with a history of sexual abuse: Risk outcome and protective factors. Journal of Interpersonal Violence, 11, 503-518.

Cinq-Mars, C., Wright, J., Cyr, M. \& McDuff, P. (2004). Sexual atrisk behaviors of sexually abused adolescent girls. Journal of Child Sexual Abuse, 12, 1-18.

Cohen, J. B., Deblinger, E., Maedel, A. B. \& Stauffer, L. B. (1999). Examining sex-related thoughts and feelings of sexually abused and nonabused children. Journal of Interpersonal Violence, $14,701-712$

Cole, J., Sprang, G., Lee, R. \& Cohen, J. (2016). The trauma of commercial sexual exploitation of youth: A comparison of CSE victims to sexual abuse victims in a clinical sample. Journal of Interpersonal Violence, 31, 122-146.

Cosentino, C. E., Meyer-Bahlburg, H. F., Alpert, J. L., Weinberg, S. L. \& Gaines, R. (1995). Sexual behavior problems and psychopathology symptoms in sexually abused girls. Journal of the American Academy of Child \& Adolescent Psychiatry, 34, 1033 1042.

Cunningham, R. M., Stiffman, A. R., Doré, P. \& Earls, F. (1994). The association of physical and sexual abuse with HIV risk behaviors in adolescence and young adulthood: Implications for public health. Child Abuse \& Neglect, 18, $233-245$.

Dillard, R., Maguire-Jack, K., Showalter, K., Wolf, K. G. \& Letson, M. M. (2019). Abuse disclosures of youth with problem sexualized behaviors and trauma symptomology. Child Abuse \& Neglect, $88,201-211$

Dong, M., Anda, R. F., Dube, S. R., Giles, W. H., \& Felitti, V. J. (2003). The relationship of exposure to childhood sexual abuse to other forms of abuse, neglect, and household dysfunction during childhood. Child abuse \& neglect, 27, 625-639.

Esparza, D. V. \& Esperat, M. C. R. (1996). The effects of childhood sexual abuse on minority adolescent mothers. Journal of Obstetric, Gynecologic \& Neonatal Nursing, 25, $321-328$.

Feiring, C., Simon, V. A. \& Cleland, C. M. (2009). Childhood sexual abuse, stigmatization, internalizing symptoms, and the deve- 
lopment of sexual difficulties and dating aggression. Journal of Consulting and Clinical Psychology, 77, 127-137.

Feiring, C., Taska, L. \& Lewis, M. (1998). The role of shame and attributional style in children's and adolescents' adaptation to sexual abuse. Child Maltreatment, 3, 129-142.

Feiring, C., Taska, L. \& Lewis, M. (1999). Age and gender differences in children's and adolescents' adaptation to sexual abuse. Child Abuse \& Neglect, 23, 115-128.

Fergusson, D. M., Horwood, L. J. \& Lynskey, M. T. (1996). Childhood sexual abuse and psychiatric disorder in young adulthood: II. Psychiatric outcomes of childhood sexual abuse. Journal of the American Academy of Child \& Adolescent Psychiatry, 35, $1365-$ 1374.

Finkelhor, D. \& Browne, A. (1985). The traumatic impact of child sexual abuse: A conceptualization. American Journal of Orthopsychiatry, 55, $530-541$.

Fiscella, K., Kitzman, H. J., Cole, R. E., Sidora, K. J. \& Olds, D. (1998). Does child abuse predict adolescent pregnancy? Pediatrics, 101, 620-624.

Friedrich, W. N., Jaworski, T. M., Huxsahl, J. E. \& Bengtson, B. S. (1997). Dissociative and sexual behaviors in children and adolescents with sexual abuse and psychiatric histories. Journal of Interpersonal Violence, 12, 155-171.

Friedrich, W. N., Urquiza, A. J. \& Beilke, R. L. (1986). Behavior problems in sexually abused young children. Journal of Pediatric Psychology, 11, $47-57$.

Garland, R. J. \& Dougher, M. J. (1990). The abused/abuser hypothesis of child sexual abuse: A critical review of theory and research. In J. R. Feierman (Ed.), Pedophilia (pp. 488 -509). New York, NY: Springer.

Hall, D. K., Mathews, F. \& Pearce, J. (1998). Factors associated with sexual behavior problems in young sexually abused children. Child Abuse \& Neglect, 22, 1045-1063.

Houck, C. D., Nugent, N. R., Lescano, C. M., Peters, A. \& Brown, L. K. (2010). Sexual abuse and sexual risk behavior: Beyond the impact of psychiatric problems. Journal of Pediatric Psychology, 35, $473-483$.

Kavemann, B. \& Lohstöter, I. (1985). Plädoyer für das Recht von Mädchen auf sexuelle Selbstbestimmung. In B. Kavemann, I. Lohstäter, L. Pagenstecher, M. Jaeckel, J. Brauckmann, E. Haarbusch \& K. Jochens, Sexualität - Unterdrückung statt Entfaltung (Alltag und Biographie von Mädchen, Bd. 9, S. 9 - 94). Wiesbaden: VS Verlag für Sozialwissenschaften.

Kendall-Tackett, K. A., Williams, L. M. \& Finkelhor, D. (1993). Impact of sexual abuse on children: A review and synthesis of recent empirical studies. Psychological Bulletin, 113, 164-180. https://doi.org/10.1037/0033-2909.113.1.164

Kwako, L. E., Noll, J. G., Putnam, F. W. \& Trickett, P. K. (2010). Childhood sexual abuse and attachment: An intergenerational perspective. Clinical Child Psychology and Psychiatry, 15, 407 422.

Mathews, B. \& Collin-Vézina, D. (2019). Child sexual abuse: Toward a conceptual model and definition. Trauma, Violence \& Abuse, 20, $131-148$.

McClellan, J., McCurry, C., Ronnei, M., Adams, J., Eisner, A. \& Storck, M. (1996). Age of onset of sexual abuse: Relationship to sexually inappropriate behaviors. Journal of the American Academy of Child \& Adolescent Psychiatry, 35, 1375-1383.

Moher, D., Liberati, A., Tetzlaff, J. \& Altman, D. G. (2009). Preferred reporting items for systematic reviews and meta-analyses: The PRISMA statement. Annals of Internal Medicine, 151, 264- 269.

Nagy, S., Adcock, A. G. \& Nagy, M. C. (1994). A comparison of risky health behaviors of sexually active, sexually abused, and abstaining adolescents. Pediatrics, 93, 570-575.

Noell, J., Rohde, P., Ochs, L., Yovanoff, P., Alter, M. J., Schmid, S., Bullard, J. \& Black, C. (2001). Incidence and prevalence of chlamydia, herpes, and viral hepatitis in a homeless adolescent population. Sexually Transmitted Diseases, 28, 4-10. https:// doi.org/10.1097/00007435-200101000-00003

Noll, J. G., Guastaferro, K., Beal, S. J., Schreier, H. M., Barnes, J., Reader, J. M. \& Font, S. A. (2019). Is sexual abuse a unique predictor of sexual risk behaviors, pregnancy, and motherhood in adolescence? Journal of Research on Adolescence, 29, 967 983.

Noll, J. G. \& Shenk, C. E. (2013). Teen birth rates in sexually abused and neglected females. Pediatrics, 131, e1181-e1187.

Noll, J. G., Trickett, P. K. \& Putnam, F. W. (2000). Social network constellation and sexuality of sexually abused and comparison girls in childhood and adolescence. Child Maltreatment, 5, 323 337.

Noll, J. G., Trickett, P. K. \& Putnam, F. W. (2003). A prospective investigation of the impact of childhood sexual abuse on the development of sexuality. Journal of Consulting and Clinical Psychology, 71, 575-586. https://doi.org/10.1037/0022-006X. 71.3.575

Ochen, A. M., Chi, P. C. \& Lawoko, S. (2019). Predictors of teenage pregnancy among girls aged 13-19 years in Uganda: A community based case-control study. BMC Pregnancy and Childbirth, 19, 211.

Orr, D. P. \& Downes, M. C. (1985). Self-concept of adolescent sexual abuse victims. Journal of Youth and Adolescence, 14, 401 410.

Paolucci, E. O., Genuis, M. L. \& Violato, C. (2001). A meta-analysis of the published research on the effects of child sexual abuse. The Journal of Psychology, 135, 17-36.

Pierre, N., Shrier, L. A., Emans, S. J. \& DuRant, R. H. (1998). Adolescent males involved in pregnancy: Associations of forced sexual contact and risk behaviors. Journal of Adolescent Health, 23, 364-369.

Pritchard, C. \& Williams, R. (2010). Comparing possible 'ChildAbuse-Related-Deaths' in England and Wales with the major developed countries 1974-2006. Signs of progress? British Journal of Social Work, 40, $1700-1718$.

Raj, A., Silverman, J. G. \& Amaro, H. (2000). The relationship between sexual abuse and sexual risk among high school students: Findings from the 1997 Massachusetts Youth Risk Behavior Survey. Maternal and Child Health Journal, 4, 125-134.

Rosario, M., Schrimshaw, E. W. \& Hunter, J. (2008). Predicting different patterns of sexual identity development over time among lesbian, gay, and bisexual youths: A cluster analytic approach. American Journal of Community Psychology, 42, 266 - 282.

Rotheram-Borus, M. J., Mahler, K. A., Koopman, C. \& Langabeer, K. (1996). Sexual abuse history and associated multiple risk behavior in adolescent runaways. American Journal of Orthopsychiatry, 66, $390-400$.

Saewyc, E. M., Magee, L. L. \& Pettingell, S.E. (2004). Teenage pregnancy and associated risk behaviors among sexually abused adolescents. Perspectives on Sexual and Reproductive Health, 36, 98-105.

Saewyc, E. M., Taylor, D., Homma, Y. \& Ogilvie, G. (2008). Trends in sexual health and risk behaviours among adolescent students in British Columbia. The Canadian Journal of Human Sexuality, $17,1$.

Santerre-Baillargeon, M., Vézina-Gagnon, P., Daigneault, I., Landry, T. \& Bergeron, S. (2017). Anxiety mediates the relation between childhood sexual abuse and genito-pelvic pain in adolescent girls. Journal of Sex \& Marital Therapy, 43, 774- 785.

Simon, V. A. \& Feiring, C. (2008). Sexual anxiety and eroticism predict the development of sexual problems in youth with a history of sexual abuse. Child Maltreatment, 13, 167-181.

Thrane, L. E. \& Chen, X. (2012). Impact of running away on girls' pregnancy. Journal of Adolescence, 35, 443-449. 
Trent, M., Clum, G. \& Roche, K. M. (2007). Sexual victimization and reproductive health outcomes in urban youth. Ambulatory Pediatrics, 7, 313-316.

Vermund, S. H., Alexander-Rodriquez, T., Macleod, S. \& Kelley, K. F. (1990). History of sexual abuse in incarcerated adolescents with gonorrhea or syphilis. Journal of Adolescent Health Care, $11,449-452$.

Whitaker, D. J., Le, B., Hanson, R. K., Baker, C. K., McMahon, P. M., Ryan, G. et al. (2008). Risk factors for the perpetration of child sexual abuse: A review and meta-analysis. Child Abuse \& Neglect, 32, 529-548.

Widom, C. \& Kuhns, J. (1996). Childhood victimization and subsequent risk for promiscuity, prostitution, and teenage pregnancy. A prospective study. American Journal of Public Health, 86, $1607-1612$.

Wilson, H. W., Emerson, E., Donenberg, G. R. \& Pettineo, L. (2013). History of sexual abuse and development of sexual risk behavior in low-income, urban african american girls seeking mental health treatment. Women \& Health, 53, 384-404.

Wirtz, U. (2001). Seelenmord: Inzest und Therapie. Freiburg: Kreuz. Worku, D., Gebremariam, A. \& Jayalakshmi, S. (2006). Child sexual abuse and its outcomes among high school students in southwest Ethiopia. Tropical Doctor, 36, 137-140.

World Health Organization (WHO). (2003). Guidelines for medicolegal care for victims of sexual violence. Verfügbar unter: http:// www.who.int/violence_injury_prevention/publications/violen $\mathrm{ce} / \mathrm{med}$ _leg_guidelines

Wu, Z. H., Berenson, A. B. \& Wiemann, C. M. (2003). A profile of adolescent females with a history of sexual assault in Texas: Familial environment, risk behaviors, and health status. Journal of Pediatric and Adolescent Gynecology, 16, 207 - 216.

Zetterqvist, M., Svedin, C. G., Fredlund, C., Priebe, G., Wadsby, M. \& Jonsson, L. S. (2018). Self-reported nonsuicidal self-injury (NSSI) and sex as self-injury (SASI): Relationship to abuse, risk behaviors, trauma symptoms, self-esteem and attachment. Psychiatry Research, 265, 309-316.

\section{Förderung}

Open Access-Veröffentlichung ermöglicht durch die PhilippsUniversität Marburg.

\section{Katharina Louisa Schulte}

Philipps-Universität Marburg

Fachbereich Psychologie

Gutenbergstraße 18

35037 Marburg

katharina.schulte@uni-marburg.de 